\title{
Adaptive Time Slotted Channel Hopping for Wireless Sensor Networks
}

\author{
Peng Du \\ Dept. of Computer Science and Information Systems \\ Birkbeck, University of London \\ Malet Street, London WC1E 7HX \\ Email: peng@dcs.bbk.ac.uk
}

\author{
Dr. George Roussos \\ Dept. of Computer Science and Information Systems \\ Birkbeck, University of London \\ Malet Street, London WC1E 7HX \\ Email: gr@dcs.bbk.ac.uk
}

\begin{abstract}
The performance of wireless sensor networks (WSN) is prone to adverse influences from a number of factors such as the interference from co-located wireless systems utilising the same spectral space. Channel hopping technique was proposed to mitigate the problem via periodic change of the operating frequency, and has been adopted in the form of time slotted channel hopping (TSCH) by IEEE 802.15.4e standard. This paper proposes adaptive slotted channel hopping (A-TSCH), an enhanced version of the TSCH aided by blacklisting technique. Complete design and implementation specifics are provided; and the results of experiments are analysed to show its advantages over existing TSCH. The main finding of this work is that ATSCH can significantly improve the reliability of channel hopping scheme and thus provide better protection from interference for wireless sensor networks.
\end{abstract}

\section{INTRODUCTION}

The proliferation of wireless communications has led to intensified utilisation of the $2.4 \mathrm{GHz}$ Industrial Scientific and Medical (ISM) band [1]. Such a phenomenon poses a challenge to wireless sensor networks (WSNs) because of their constrained capacity to contend with more powerful equipment for scarce spectrum. The channel hopping technique addresses the coexistence issue [2], [3] through periodical switch of the communication frequency, which distributes the risk of encountering interference over all available channels [4]. The technique has been widely accepted [5], [6] and time slotted channel hopping (TSCH) is now part of the forthcoming IEEE 802.15.4e [7].

One of the limitations of TSCH stems from the fact all 16 IEEE 802.15.4 channels are used indiscriminately, whereas channels often experience different level of interference. [8] suggests that a technique known as "blacklisting" can alleviate such inefficiency, based on a posteriori statistical arguments. The principle of the scheme is to use a selected subset of "high quality" spectrum rather than the entirety of channels, i.e. avoid using heavily interfered frequencies. Despite being embraced by a number of technologies [5], [6], no implementation of blacklisting has been specified.

We in this paper propose the adaptive time slotted channel hopping (A-TSCH) algorithm. The main contributions are threefold. First, the A-TSCH algorithm is designed by employing blacklisting technique; secondly, an implementation on wireless sensor network platform is provided and tested with various configurations applied; and thirdly the experimental evidences are analysed, substantiating the improved reliability and the correctness of the algorithm.

The remainder of the paper is composed as follows: Section II reviews some of the relevant studies on channel hopping and blacklisting. The motivations and reasoning of A-TSCH are discussed in Section III. The algorithm is then described in detail in Section IV. After a summary of experiment settings in Section V, Section VI presents an analysis of experimental results and a discussion of findings. And finally in Section VII our conclusions are drawn.

\section{RELATED WORK}

The channel hopping technique has been embraced by a number of technologies and standards. A one of the most notable example, the IEEE 802.15.4e Task Group [7] adopts the time slotted channel hopping (TSCH) technique to enhanced the existing IEEE 802.15.4 standard.

TSCH stems from the Time Synchronized Mesh Protocol (TSMP) [4]. The time space is divided into a series of infinite number of discrete slots and every node in the network maintains a synchronised record of the number of elapsed slots. Using this number as the sole input to an equation known by all, operating channels in each slot is calculated. Communications thus randomly take place in random channels, which reduces the influence of interference as well as multi-path fading since the risk is spread over all channels [8].

The concept of channel hopping can also be found in Bluetooth and WirelessHART [6]. Bluetooth devices constantly switch among $791-\mathrm{MHz}$ wide channels to improve the coexistence with technologies such as the IEEE 802.11 [5]. WirelessHART, on the other hand, is an open-standard technology based on TSMP for IEEE 802.15.4 sensors. 
A possible enhancement to $\mathrm{TSCH}$ is revealed in [8]. Connectivity traces collected in experiments conducted beforehand are replayed. Although the study mainly aims to demonstrate the advantage of channel hopping in general over single-channel scheme, it finds improved packet delivery ratio (PDR) if certain channels are removed from the channel hopping sequence. So it argues that by excluding some channels of low quality the overall performance of $\mathrm{TSCH}$ can be improved. However the work is purely statistical and provides no suggestion on algorithm. The tactic of channel hopping is also upheld by Bluetooth and WirelessHART [9], which respectively provide advanced functionality known as adaptive frequency hopping (AFH) [5] and Blacklisting [10]. However, mechanism of blacklisting decision is not specified in any of the above standards.

Inspirations can be drawn from slightly different areas too. The dynamic hopping MAC (DH-MAC) protocol is proposed in [11] for secondary users in the licensed bands where the availability of frequencies depend on the behaviours of primary users (PUs). Motes periodically hop to a random frequencies and, if it is being used by PUs, skip to the next one in the hopping sequence. Because the effect of PUs on DH-MAC can be viewed as comparable to powerful interference which paralyses certain WSN channels, this protocol is to some extent analogous to a scheme that helps wireless sensor networks (WSNs) avoid severely affected channels, and the benefit should still hold for WSNs in 2.4 $\mathrm{GHz}$.

\section{Motivations And Rationales}

Because of the minimal regulations on the $2.4 \mathrm{GHz}$ ISM band, devices may arbitrarily access any part of the spectrum [12], which results in the diversity of utilisation profiles and, accordingly, levels of interference in the channels [13].

Wireless sensors are particularly vulnerable to interferences since they often come with limited transmission power compared with powerful $2.4 \mathrm{GHz}$ devices such Wi-Fi routers and microwave ovens. Although the TSCH technique mentioned earlier is able to abate such threats by constantly switching to random channels, it does not differentiate the channel qualities and consequently communications inevitably take place in those heavily utilised frequencies at some point. Our aim is to design a channel access algorithm that excludes the undesirable channels from the hopping sequence, a practise known as Blacklisting, in order to improve the reliability of communications whilst preserving the benefit of TSCH.

One of the most fundamental requirements is the strategies of channel quality estimation, based on which the improved channel hopping sequence can be established. The percentage of successful transmission over a test-window of sent packets [14], usually referred to as packet delivery ratio (PDR), has

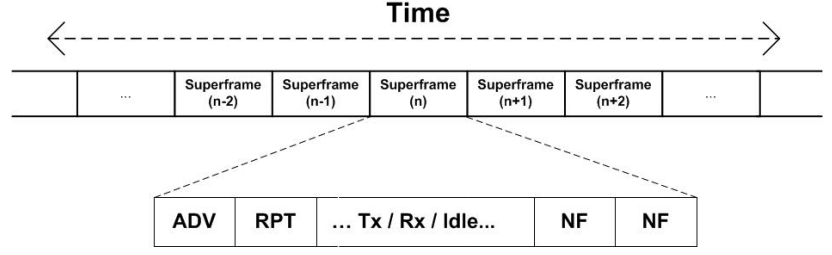

Fig. 1. The time space is divided into superframes, each consisting of a fixed number of slots. In our implementation there are 5 types of timeslots excluding idle type, i.e. 3 from TSCH (ADV, Tx and $\mathrm{Rx}$ ) and 2 additional for A-TSCH (RPT and NF)

been frequently adopted as the quality indicator in past studies [15]-[18]. For example in [15], the PDR of the operating channel is constantly monitored and updated. As soon as it drops to a certain threshold the channel is classified as undesirable and the system switches to another frequency. Such an approach, nevertheless, has several disadvantages. On one hand, if the window is too small, the test outcomes of individual packets carry exaggerated influences and the resulting PDR can be very unsteady; on the other hand, too large a test-window size could lead to the latency in capturing and adapting to the dynamic spectral condition [14].

Spectrum sensing [19], [20] provides an alternative to channel estimation. Specifically, by detecting the ambient energy level, which we in the future refer to as the noise floor, the intensity of channel utilisation can be gauged [21] as noise floors exhibit a very linear function of input power [22], [23]. Our work employs this method to assess the channel qualities. Despite the modest conceptual complexity, the method does pose a practical difficulty since communications must be suspended during noise floors listening [24]. Fortunately, such requirement can be relatively easily met thanks to the infrastructure of $\mathrm{TSCH}$. More details are provided in following sections.

\section{A-TSCH ALgORITHM}

In this paper we propose the adaptive time slotted channel hopping (A-TSCH) algorithm, which provides enhanced reliability on the basis of TSCH technique of IEEE 802.15.4e standard [7]. The distinctive characteristic of A-TSCH is its ability to hop selectively among a subset of channels considered "reliable", unlike the IEEE 802.15.4e TSCH which indiscriminately uses all 16 channels in the $2.4 \mathrm{GHz}$ band. The rest of this section begins with an elaborated description of TSCH; and afterwards the A-TSCH algorithm is detailed following the logical phases of its functionality.

\section{A. IEEE 802.15.4e TSCH}

Since our algorithm is designed as an improved version of $\mathrm{TSCH}$, it is useful to look into more details of the original scheme. 
TSCH divides time into endless successive slots. Furthermore, every fixed number of slots are grouped as a superframe. Thus the time space can also be interpreted as a series of consecutive superframes consisting of constant number of slots, as illustrated in Figure 1. Slots are appointed different types depending on their positions within superframes. TSCH uses 3 kinds of slots excluding the "idle" type: advertisement (ADV), transmit (Tx) and receive (Rx).

ADV slots are reserved for the broadcast of advertisements containing topology and timing information. Once the ADV packets have been successfully propagated, all nodes share the identical $A S N$ and their superframes are aligned, and then able to communicate in $\mathrm{Tx}$ and $\mathrm{Rx}$ slots. The topology of a synchronised TSCH network conform to Destination Oriented Directed Acyclic Graph (DODAG) where every node select one of its neighbours closer to the WSN gateway as its routing parent and in the future only accept ADV packets from this particular node until the next routing update. TSCH schedules that there is at most one pair of sender and receiver communicating in any given $\mathrm{Tx}$ or Rx slot, so that member nodes do not interfere with each other.

Key to the scheme is a variable called absolute slot number $(A S N)$ which holds the number of elapsed timeslots. From the $A S N$ nodes can deduce the current slot type and hence the associated operations. Moreover, channels used in slot are computed using $A S N$ with the following equation.

$$
\begin{gathered}
\text { Channel }=A S N \% 16+11 \\
A S N \in[0, \infty)
\end{gathered}
$$

It can be derived from equation (1) that Channel $\in[11,26]$ and therefore all $162.4 \mathrm{GHz}$ channels (number 11 to 26) are accessed indiscriminately. Thus sometimes the TSCH of IEEE $802.15 .4 \mathrm{e}$ is referred to as a "blind" channel hopping scheme.

\section{B. Channel Sampling}

It is mentioned earlier that channel sampling entails the suspension of communication [21]. Such requirement can be straightforwardly fulfilled in A-TSCH by introducing an ancillary noise floor (NF) slot type.

The algorithm regulates that no communication can take place in NF slots. Channels associated with these slots, however, are calculated as usual with equation (1) and their noise floors are collected, which in practise is achieved by accessing the Received Signal Strength Indicator (RSSI) register of the radio transceiver [24]. In the current version of A-TSCH, two NF slots are placed at the rear of every superframe as illustrated in Figure 1.

\section{Channel Quality Estimation}

The sampled noise floors are processed using simple exponential smoothing (SES) technique as shown in equation (2) to generate quality estimation $(Q E)$ for each channel.

$$
\begin{aligned}
Q E[c]_{k} & =\left\{\begin{array}{l}
N F[c]_{0} \quad \text { if } k=0 \\
\alpha Q E[c]_{k-1}+(1-\alpha) N F[c]_{k} \quad \text { if } k \in[1, \infty)
\end{array}\right. \\
c & \in[11,26], \quad \alpha \in[0,1]
\end{aligned}
$$

$Q E[c]_{k}$ denotes the quality estimation of channel $c$ obtained after the $k^{t h}$ instance of noise floor listening. The initial estimation $Q E[c]_{0}$ is assigned $N F[c]_{0}$, the very first noise floor reading. As per equation (2), subsequent estimations at $k$ are calculated using previous records $Q E[c]_{k-1}$ and most recent noise floor $N F[c]_{k}$, subject to coefficient $\alpha$.

\section{Blacklisting}

A blacklist records the channels excluded from the hopping sequence. Various criteria may be adopted in making blacklist decisions based on the quality estimations described above. For instance, the algorithm can blacklist a pre-defined number of channels with the poorest estimated quality or alternatively, any channels falling short of certain standards.

In A-TSCH it is crucial that nodes maintain the knowledge of neighbours' blacklists so that senders and receivers use the identical hopping sequences when communicating. For that purpose, nodes insert their local blacklist into the ADV packets therefore the blacklists are propagated in ADV slots following a similar process as the synchronization of timing information. Because of the top-down hierarchy of ADV synchronisation defined by TSCH routing process, the blacklist is disseminate downwards from the WSN gateway node.

\section{E. Link-Mask Generation}

The mechanism described above only enables unidirectional blacklist disseminations, as employed in our previous work [24]. However, the spectral condition exhibits spatial variability [25] and accordingly it is possible that channel qualities observed by the senders and receivers do not match. Consequently channel hopping sequence determined by both ends of the link make more sense, in other words, links rather than either of the communicating sides alone should dictate the hopping sequence in A-TSCH. We term such link-associated blacklists as link-masks for distinction with the local blacklists of individual nodes, and the definition is given in equation (3).

$$
L M_{l, n}=B L_{l} \cap B L_{n}
$$


$B L_{l}$ and $B L_{n}$ denote the local blacklist of node $l$ and its scheduled communication partner node $n$, respectively. The link-mask $L M_{l-n}$ is then defined as the intersection of both blacklists.

Nodes must be able to compute the link-masks for each communication and consequently the blacklist propagation needs to be extended to bi-directional. To facilitate such operations, A-TSCH introduces an additional auxiliary report (RPT) slot type as illustrated in Figure 1. Within RPT slots, nodes construct RPT messages containing local blacklist and transmitted them to their upstream neighbour via unicast. This mechanism, together with the ADV packet dissemination, ensures the blacklists are propagated both upwards and downwards in the network hierarchy.

In $\mathrm{Tx}$ or Rx slots, communicating nodes firstly calculate channel with equation (1). The generated channel is then checked against the link-mask computed with equation (3). Sending or receiving are performed normally if the channel is not blacklisted; otherwise a new channel candidate is randomly generated and checked. The process is repeated until a non-blacklisted channel is acquired.

\section{EXPERIMENTS}

Experiments were conducted in an open office space where typically an excess of 10 WLANs and Bluetooth devices are present. A-TSCH is coded on top of Berkeley's OpenWSN stack [26] and the implementation is installed in Guidance and Inertial Navigation Assistant (Gina) motes [27]. Note the choice of hardware is purely in the interest of convenience and the algorithm should apply to other platforms such as TMote Sky [28] used in our previous work [24]. One of the motes was connected to an IPv6 gateway router and thus acted as the WSN gateway. Other nodes were programmed to transmit 300 packets to the gateway at $0.5 \mathrm{~Hz}$.

In order to examine blacklisting criteria described in Section IV.D, experiments were carried out with different configurations as listed in column (A) to (E) of Table I. Experiments of each setting was repeated 6 times so a total of 30 tests took place. Configuration (A) and (E) represent two extreme cases. A-TSCH with blacklist of size zero in (A) is equivalent to blind TSCH because no channel can be blacklisted. In contrast, (E) allows the exclusion of any number of channels provided their noise floors exceed the threshold of $-87 \mathrm{dBm}$ which is based on the findings in [29]; a pre-defined "always on" channel is protected to ensure at least one of the channels is available.

\section{ANALYSIS}

In this section the performance of A-TSCH are analysed and the verifications of our blacklisting process is provided too. For the convenience of analysis, we focus on data
TABLE I

BLACKLIST SIZE CONFIGURATIONS

\begin{tabular}{|c||c|c|c|c|c|}
\hline Experiment Setting & A & B & C & D & E \\
\hline Size of blacklist & 0 & 3 & 6 & 9 & $\begin{array}{c}\text { Semi-controlled: based on } \\
\text { pre-defined threshold, can } \\
\text { blacklist up to 15 } \\
\text { channels }\end{array}$ \\
\hline
\end{tabular}

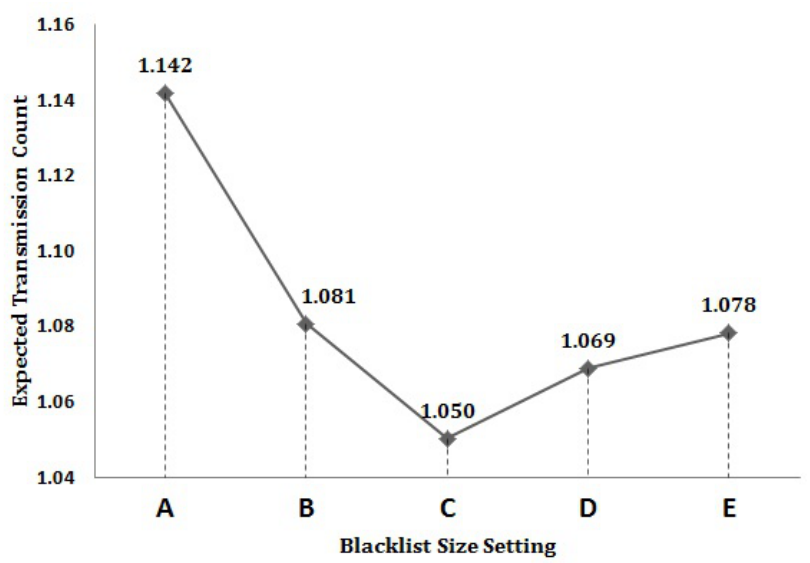

Fig. 2. The Expected Transmission Count (ETX) observed in experiments (A) through (E). Data points are shown as filled diamonds with corresponding ETX values. It is apparent that the A-TSCH leads to a lower chance of packet loss in (B) through (E) compared to that of (A) where blind TSCH was used

collected from the gateway and another specific node.

To quantify the reliability of communication, expected transmission count (ETX), defined as $\frac{1}{P D R}$ [30], is calculated for all settings (A) to (E) and illustrated in Figure 2. ETXs are multiplicative inverse values of packet delivery ratio (PDR), and therefore represent the average numbers of attempts for transmissions to be successful [8].

The mean ETXs achieved in all tests are shown in Figure 2 beside the diamond-shaped markers. It is observed that experiment with blacklisting-enabled settings (B - E) uniformly delivered lower ETXs than experiment (A) which used blind TSCH. Statistics are also provided in Table II, showing improvement of at least 5.6\%. The reduced ETXs proves that A-TSCH is able to improve the communication reliability by filtering out heavily utilised frequencies, though no sufficient evidence are available to derive deterministic relationships between the performance and the blacklist size.

To further demonstrate that positive effect of A-TSCH, we have traced the momentary PDR values upon receipts of each packet during the communications and compiled such fluctuations in Figure 3, where 3(a) and 3(b) illustrate series of PDR data monitored with setting (A) and (B - E), respectively.

Albeit the relationship between the two does not seem to clear at the beginning, the series in 3(b) starts to show 
TABLE II

STATISTICAL COMPARISON OF ETXS

\begin{tabular}{|c||c|c|c|c|c|}
\hline Experiment Setting & A & B & C & D & E \\
\hline Average ETX & 1.142 & 1.081 & 1.050 & 1.069 & 1.078 \\
\hline Improvement & N/A & $5.3 \%$ & $8.1 \%$ & $6.4 \%$ & $5.6 \%$ \\
\hline
\end{tabular}

The average ETXs are at least 5.6\% lower when blacklist is enabled (B) through (E). This indicates A-TSCH increase the chance of successful transmissions as the noisy channels are avoided

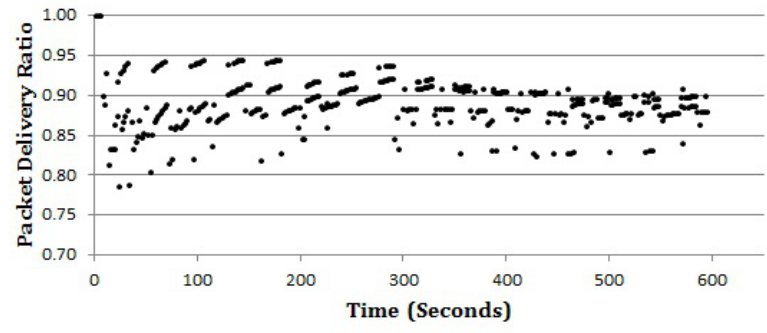

(a)

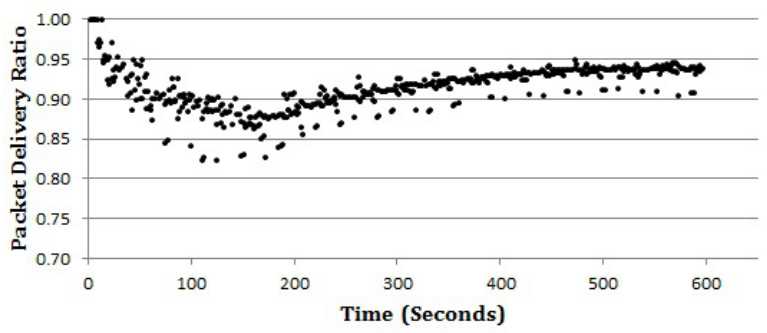

(b)

Fig. 3. Average real-time PDR of (a) blind channel hopping and (b) A$\mathrm{TSCH}$. The figure shows blacklisting results in not only stable and higher performance but also a growing tendency of PDR.

superiority after approximately 200 seconds since the commencement of experiments, as the delivery ratio is dominantly above 0.9 compared with the scattered values in 3(a). Moreover, 3(b) shows a steady trend of growth whilst 3(a) displays a different tendency. This is because blacklist decisions become increasingly accurate as continuing communications allows for more noise floor readings to be collected. And the more precise blacklisting are the better protections from interfered channels A-TSCH provides.

Whilst the improved reliability is visualised in Figure 2 and 3 , Figure 4 verifies the correctness of the blacklist algorithm of A-TSCH. The average possibility that channel $c$ was chosen to be blacklisted, $B R[c]$ as defined in equation (4), is used to quantify how likely a the channel is to be considered "noisy".

$$
\begin{aligned}
B R & {[c]=\frac{\text { how many times channel } \mathrm{c} \text { is blacklisted }}{\text { total number of blacklist decisions }} } \\
c & \in[11,26]
\end{aligned}
$$

It is obvious in Figure 4(a) that channel range $\{16-19\}$ and $\{21-24\}$ are more frequently blacklisted by a large scale compared with rest. Because our implementation of
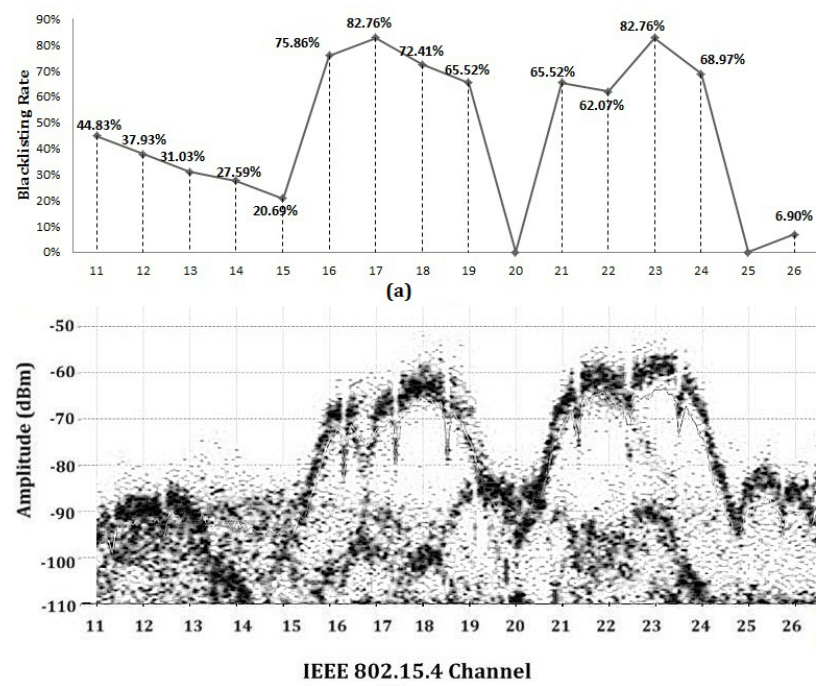

(b)

Fig. 4. (a) The Blacklisting Rate (BR) of all 16 channels and (b) Noise floors of IEEE 802.15.4 channels captured with Wi-Spy.

A-TSCH depend solely on the level of ambient energy to make blacklist decisions, the result effectively indicates the fact that notably high noise floors were witnessed in these channels.

Wi-Spy, a commercial spectrum analyser [31] was used to provide confident knowledge of channel utilisation profiles. It was placed near the pair of studied nodes during the experiments and the result of capture noise floor level are displayed in Figure 4(b). It can be seen that channel $16-19\}$ and $\{21-24\}$ are associated with conspicuously high noise levels of at least $-70 \mathrm{dBm}$, whilst for the rest of the $2.4 \mathrm{GHz}$ channels the averages are approximately $-90 \mathrm{dBm}$. Such a contrast is in accordance with the findings of $B R \mathrm{~s}$ in $4(\mathrm{a})$, which demonstrate that our algorithm is able to accurately estimate the intensity of ambient noise and accordingly identify and blacklist heavily utilised channels.

\section{CONCLUSION}

In this paper, we examined the time slotted channel hopping (TSCH) of IEEE 802.15.4e and suggested further protection against interference via excluding channels of high noise levels from the hopping sequence, a technique known as blacklisting [8]. The adaptive time slotted channel hopping (A-TSCH) algorithm was then proposed to embody such a concept. Under the scheme spectral conditions at both of the senders and receivers are taken into account to formulate the desirable channel hopping sequence. The algorithm was implemented and tested with different configurations. Experimental evidences demonstrated improved chances of successful transmissions and over the blind IEEE 802.15.4e $\mathrm{TSCH}$. The accuracy of blacklisting was also verified using a 
commercial spectrum analyser.

\section{REFERENCES}

[1] S. Pollin, M. Ergen, A. Dejonghe, L. V. D. Perre, F. Catthoor, I. Moerman, and A. Bahai, "Distributed cognitive coexistence of 802.15.4 with 802.11," in 1st Int. Conf. Cognitive Radio Oriented Wireless Networks and Comm., 2006.

[2] H. Khaleel, C. Pastrone, F. Penna, M. Spirito, and R. Garello, "Impact of Wi-Fi traffic on the IEEE 802.15.4 channels occupation in indoor environments," in Int. Conf. Electromagnetics in Advanced Applications, 2009. ICEAA '09., 2009, pp. 1042 - 1045.

[3] C. M. D. Dominicis, P. Ferrari, A. Flammini, E. Sisinni, M. Bertocco, G. G. adn C Narduzzi, and F. Tramarin, "Investigating WirelessHART coexistence issues through a specifically designed simulator," in Instrumentation and Measurement Technol. Conf. 2009 IEEE. IEEE, 2009, pp. 1085-1090.

[4] K. S. J. Pister and L. Doherty, "TSMP: Time Synchronized Mesh Protocol," in Parallel and Distributed Computing Systems, 2008.

[5] Bluetooth Core Specifications version 4.0, SIG Bluetooth Std.

[6] "WirelessHART technical data sheet."

[7] IEEE 802.15 WPA Task Group 4e (tg4e) website. [Online]. Available: http://www.ieee802.org/15/pub/TG4e.html

[8] T. Watteyne, A. Mehta, and K. Pister, "Reliability through frequency diversity: why channel hopping makes sense," in Proc. 6th ACM PEWASUN. ACM, 2009, pp. 116-123.

[9] D. Christin, P. S. Mogre, and M. Hollick, "Survey on wireless sensor network technologies for industrial automation: The security and quality of service perspectives," Future Internet, vol. 2, no. 2, pp. 96-125, 2010. [Online]. Available: http://www.mdpi.com/1999-5903/2/2/96

[10] Co-existence of WirelessHART with other wireless technologies. [Online]. Available: http://www.hartcomm.org/protocol/training/resources/ wiHART_resources/

[11] C.-F. Shih, T. Y. Wu, and W. Liao, "Dh-mac: A dynamic channel hopping mac protocol for cognitive radio networks," in Communications (ICC), 2010 IEEE International Conference on, may 2010, pp. 1 -5.

[12] C. T. Ee, "Interference avoidance in wireless multihop networks," poster session presented at the 2nd Annu. IEEE Commun. Soc. Conf. Sensor and Ad Hoc Commun. and Networks, 2005.

[13] Y. Liu, "Performance improvement of wireless communications using frequency hopping spread spectrum," Int. J. Commun., Network and System Sci., vol. 3, pp. 805-810, Oct. 2010.

[14] M. Senel, K. Chintalapudi, D. Lal, A. Keshavarzian, and E. Coyle, "A kalman filter based link quality estimation scheme for wireless sensor networks," in Global Telecommunications Conference, 2007. GLOBECOM '07. IEEE, nov. 2007, pp. $875-880$.

[15] B. Kerkez, T. Watteyne, M. Magliocco, S. Glaser, and K. Pister, "Feasibility analysis of controller design for adaptive channel hopping," in Proc. 4th VALUETOOLS. ICST, 2009, pp. 76:1-76:6.

[16] R. Tomasi, H. Khaleel, F. Penna, C. Pastrone, R. Garello, and M. A. Spirito, "Frequency agility in IPv6-based wireless personal area networks (6LoWPAN)," in WWIC'10, 2010, pp. 146-157.

[17] T. Liu and A. Cerpa, "Foresee (4c): Wireless link prediction using link features," in Information Processing in Sensor Networks (IPSN), 2011 10th International Conference on, april 2011, pp. $294-305$.

[18] B. Radunovic, A. Proutiere, D. Gunawardena, and P. Key, "Exploiting channel diversity in white spaces," Microsoft Technical Report, Tech. Rep., 2011.

[19] S. Haykin, "Cognitive radio: Brain-empowered wireless communications," IEEE J. Sel. Areas Commun., vol. 23, pp. 201-220, 2005.

[20] I. F. Akyildiz, W.-Y. Lee, M. C. Vuran, and S. Mohanty, "Next generation/dynamic spectrum access/cognitive radio wireless networks: a survey," Comput. Netw., vol. 50, no. 13, pp. 2127-2159, 2006.

[21] T. Yucek and H. Arslan, "A survey of spectrum sensing algorithms for cognitive radio applications," IEEE Commun. Surveys Tuts., vol. 11, pp. $116-130,2009$.

[22] "At86rf231/zu/zf datasheet." [Online]. Available: http://www.atmel.com/ Images/doc8111.pdf

[23] "Chipcon CC2420 datasheet." [Online]. Available: http://inst.eecs. berkeley.edu/ cs150/Documents/CC2420.pdf

[24] P. Du and G. Roussos, "Adaptive channel hopping for wireless sensor networks," in 2011 International Conference on Selected Topics in Mobile and Wireless Networking (iCOST), 2011.
[25] D. Sexton, M. Mahony, M. Lapinski, and J. Werb, "Radio channel quality in industrial wireless sensor networks," in Sensors for Industry Conference, 2005, feb. 2005, pp. $88-94$

[26] Berkeley's openwsn. [Online]. Available: http://openwsn.berkeley.edu

[27] "Warpwing project page." [Online]. Available: http://warpwing. sourceforge.net/

[28] "TMote Sky datasheet." [Online]. Available: http://www.bandwavetech. com/download/tmote-sky-datasheet.pdf

[29] K. Srinivasan and P. Levis, "RSSI is under appreciated," in Proc. 3rd Workshop on Embedded Networked Sensors (EmNets, 2006.

[30] D. S. J. D. Couto, "High-throughput routing for multi-hop wireless networks," Ph.D. dissertation, MIT, June 2004.

[31] Wi-Spy. [Online]. Available: http://www.metageek.net/products/wi-spy/ 